Rev Inv Vet Perú 2009; 20 (2): 291-296

\title{
INFECCIÓN EXPERIMENTAL DE CERDOS DE UN MES DE EDAD CON ESPORAS DE Enterozytozoon bieneusi
}

\author{
Experimental Infection of One-Month-Old Piglets with Spores of \\ ENTEROZYTOZOON BIENEUSI
}

\author{
Jéssica Alvarado G. ${ }^{1}$, Armando González Z. ${ }^{1}$, Robert Gillman ${ }^{2}$ y Teresa López U.,4
}

\section{Resumen}

La microsporidiosis humana era considerada una enfermedad exclusiva de personas VIH positivas o de pacientes inmunocomprometidos pero actualmente se sabe que afecta, además, a pacientes inmunocompetentes. El microsporidio más común es el Enterocytozoon bieneusi. El presente trabajo tuvo como objetivo demostrar la infección experimental de cerdos de un mes de edad con 840000 esporas de E. bieneusi por vía oral. Se utilizaron nueve lechones procedentes de una granja tecnificada con muy pocas probabilidades de infección a este parásito. Para el diagnóstico del parásito se utilizó la coloración tricrómica (en heces) y la coloración de plata (tejido intestinal). Se logró observar esporas de E. bieneusi en heces y en cortes de intestino de todos los lechones. El trabajo demostró que los cerdos de un mes de edad son susceptibles a la infección con esporas de E. bieneusi y, por lo tanto, este animal podría ser considerado como modelo experimental en el estudio de la microsporidiosis humana causada por E. bieneusi.

Palabras clave: microsporidiosis, Enterocytozoon bieneusi, VIH, cerdos

\section{AbSTRACT}

Human microsporidiosis has been considered a disease of the VIH positive patients or other patients with immunosupression. At present, it is known that microsporidiosis affects not only these patients but also the immunocompetent. The most common species is the Enterocytozoon bieneusi. The present study demonstrates the experimental oral infection of one-month-old piglets with spores of E. bieneusi. Nine piglets from a commercial farm with very low risk of infection by this parasite were used. Two staining techniques were utilized for the diagnosis of the parasite: Tricromic Blue Stain (in fecal samples) and Silver Stain (in intestinal wall). Spores of E. bieneusi were observed in feces

${ }^{1}$ Laboratorio de Medicina Veterinaria Preventiva, ${ }^{3}$ Laboratorio de Microbiología y Parasitología Veterinaria, Facultad de Medicina Veterinaria, Universidad Nacional Mayor de San Marcos, Lima

${ }^{2}$ Laboratorio de Enfermedades Infecciosas, Facultad de Ciencias y Filosofía, Universidad Peruana Cayetano Heredia, Lima

${ }^{4}$ E-mail:mtlu@terra.com.pe 
and intestinal sections of all animals. The estudy showed that pigs of one month of age are susceptible to the infection with spores of E. bieneusi and therefore, could be considered as experimental model in the study of the human microsporidiosis caused by E. bieneusi.

Key words: microsporidiosis, Enterocytozoon bieneusi, VIH, pig

\section{INTRODUCCIÓN}

La microsporidiosis es considerada como una enfermedad oportunista y emergente que provoca serias patologías en individuos inmunocomprometidos, especialmente en personas con SIDA (Atías, 1991). Sin embargo, también puede afectar a personas inmunosuprimidas que hayan recibido un transplante de órgano y a personas inmunocompetentes (Weber y Bryan, 1994). Tiene una gama amplia de hospedadores, tanto vertebrados como invertebrados y se encuentra distribuida en todo el mundo (Murray et al., 1997).

La enfermedad es causada por microorganismos del Phylum Microspora (OPS, 2003). Diversas especies de microsporidios se han encontrado en tejidos humanos (Encephalitozoon cuniculi, E. hellen, E. intestinalis, Brachiola vesicularum, Nosema connori, N. ocularum, Pleistophora sp., Trachipleistophora hominis, T. anthropoptera, Vittaforma corneae y Enterocytozoon bieneusi) (Weber y Bryan, 1994); siendo el Enterozytozoon bieneusi el más comúnmente diagnosticado en el humano (Dengjel et al., 2001). El E. bieneusi infecta principalmente el epitelio intestinal y causa diarrea, no solo en personas inmunocomprometidas, sino también en personas inmunocompetentes, aunque en ellas la diarrea es autolimitante (Weber y Bryan, 1994).

Se desconoce el reservorio para la infección humana por E. bieneusi, pero se considera que la transmisión se produce por ingestión de esporas procedentes de la orina o heces de animales o personas infectadas (Murray et al., 1997). En este sentido, su importancia en salud pública se ha incrementado debido a que los microsporidios que infectan a humanos han sido identificados en fuentes de agua y animales (Desplazes et al., 2000). Dada la enorme variedad de animales infectados por microsporidios, se debe tener en cuenta una posible propagación zoonótica (Cama et al., 2007).

El primer caso de microsporidiosis humana se reportó en 1985, donde se encontraron esporas de E. bieneusi en los enterocitos de un paciente VIH positivo (Desportes et al., 1985). Por otro lado, el primer reporte de microsporidiosis humana por E. bieneusi en un paciente VIH negativo se describió en 1994 y, en ese entonces, se concluyó que era un parásito natural en el hombre y que, además, podía causar enfermedad en pacientes inmunocompetentes (Sandfort et al., 1994). No obstante, estudios posteriores realizados en mujeres embarazadas, donadores de sangre, trabajadores de camales y criadores de perros, mediante la detección de anticuerpos específicos contra microsporidios, demostraron que también afecta a individuos inmunocompetentes (Del Águila et al., 2001).

En la actualidad, es muy poca la información que se tiene de la enfermedad, y la existente está basada en estudios realizados directamente en humanos. Asimismo, se vienen realizando diversos estudios para poder cultivar el E. bieneusi aunque sin éxito hasta el momento (Didier y Weiss, 2006). El objetivo del presente estudio fue lograr la infección experimental de cerdos de un mes de edad con esporas de E. bieneusi a fin de evaluar la posibilidad de usar al cerdo como 
un modelo experimental para realizar investigaciones relacionadas al ciclo biológico, vías de contaminación, comportamiento patológico en las células invadidas, y uso de drogas para su eliminación, entre otros.

\section{Materiales y MéTodos}

\section{Animales}

Se utilizaron nueve lechones de un mes de edad, procedentes de una granja de cerdos de crianza tecnificada, de $9 \mathrm{~kg}$ de peso en promedio y negativos a E. bieneusi. Los animales fueron alojados, desde su llegada y durante todo el experimento, en los corrales del Laboratorio de Medicina Veterinaria Preventiva de la Facultad de Medicina Veterinaria, Universidad Nacional Mayor de San Marcos, Lima. Desde su arribo y durante todo el experimento los animales recibieron alimento balanceado comercial y agua ad libitum.

\section{Pre-infección}

Se realizó el monitoreo de las heces durante seis días consecutivos para comprobar la negatividad de los animales a $E$. bieneusi. Tres días previos a la infección, los lechones recibieron una papilla consistente en Josten Milk, afrecho y agua, además de su alimento, y un día antes de la infección solo se les proporcionó la mitad de su ración de alimento a fin de familiarizarlos y asegurar la ingestión de la dosis infectiva.

El procesamiento del inóculo se realizó en el Laboratorio de Parasitología de la Facultad de Ciencias y Filosofía, Universidad Peruana Cayetano Heredia. Se utilizó una muestra proveniente de un niño con microsporidiosis, la cual fue homogeneizada y dividida en alícuotas de $2 \mathrm{ml}$. Luego la muestra se lavó tres veces con agua destilada y centrifugaciones a $2500 \mathrm{x}$ g durante $30 \mathrm{minu}-$ tos. Cada sedimento obtenido fue resuspendido en $2 \mathrm{ml}$ de PBS, pH 7.2-7.4, 0.01M. Para el recuento de esporas se tomó $10 \mu 1$ de la suspensión en un portaobjetos, se dejó secar al ambiente y se tiñó con coloración tricrómica (Isemberg, 1998). Se obtuvo un recuento de 4200 esporas $/ 10 \mu 1$.

\section{Infección}

El inóculo para cada lechón se preparó agregando $8 \mathrm{ml}$ de PBS, pH 7.2-7.4, 0.01M, a tubos que contenían los $2 \mathrm{ml}$ de suspensión de esporas y luego se mezcló con la papilla. Cada lechón, en ayunas y alojado en una jaula individual, fue infectado por vía oral con, aproximadamente, 840000 esporas de $E$. bieneusi.

\section{Post-infección}

Las heces de cada animal se recolectaron durante 30 días y se procesaron con el método de concentración de Ritchie y coloración tricrómica. Dos lechones se sacrificaron al día 10 post-infección (p.i.) y el resto al final del experimento. En la necropsia se tomaron muestras de duodeno, yeyuno, íleon y colon. Las muestras de tejidos fueron procesadas y teñidas con coloración de plata.

La función de la probabilidad de infecciones se calculó empleando la distribución Beta. Para graficar la distribución Beta, $\beta\left(\alpha_{1}, \alpha_{2}\right)$ se consideraron como parámetros $\alpha_{1}=$ éxitos +1 y $\alpha_{2}=$ fracasos +1 . La función de probabilidad se calculó empleando el paquete estadístico de simulación estocástica@Risk 4.5 (Palisade Corp). La simulación se corrió por 10000 iteracciones y se graficó la función de probabilidad con intervalos de confianza del $90 \%$.

\section{Resultados y Discusión}

Todos los animales infectados llegaron a eliminar esporas con las heces. El inicio de la eliminación de esporas estuvo entre los días 4-6 p.i. (Fig. 1). Este tiempo fue menor al determinado en otras infecciones experi- 


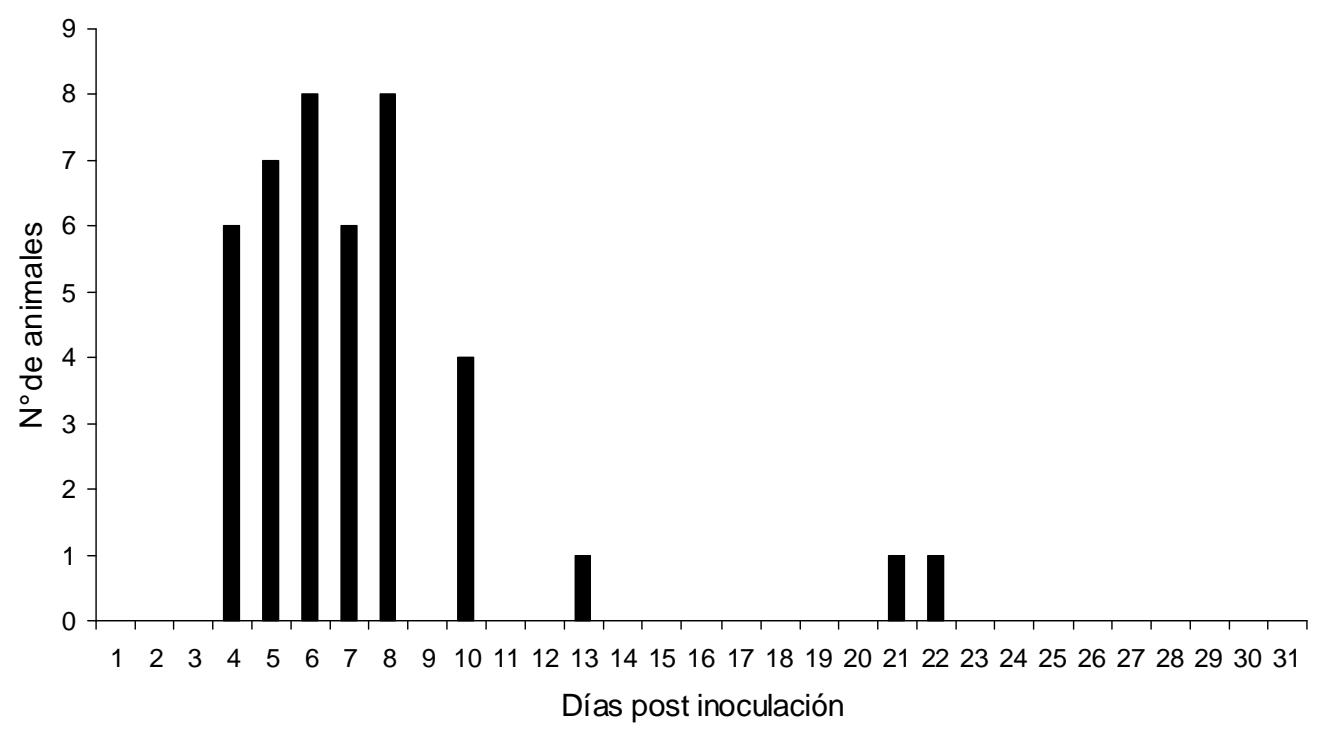

Figura 1. Número de animales con presencia de esporas de Enterocytozoon bieneusi en heces (diagnóstico con coloración tricrómica)

mentales, que estuvo entre los 9 a 12 días p.i. (Tzipori et al., 1998). Este resultado se podría deber al número mayor de esporas utilizados en el presente estudio (840 000 esporas/animal) en comparación con la dosis del inóculo empleado por el grupo de Tzipori, que fue entre 5000 a 50000 esporas/animal.

Las esporas se eliminaron por espacio de 2 a 6 días en la mayoría de los lechones. La eliminación de esporas no fue constante sino, más bien, intermitente. En este sentido, uno de los lechones eliminó esporas hasta el día 8 p.i., dejó de excretar esporas y luego continuó excretando esporas hasta el día 22 p.i., dando un total de 12 días de eliminación de esporas. Este hecho podría deberse a que E. bieneusi podría presentar estadios intracelulares en diferentes grado de desarrollo, o tal vez a una localización extra intestinal. Esta situación se observa en Isospora suis, donde la eliminación de ooquistes puede ser bifásica, debido a la reinvasión del epitelio intestinal de los estadios presentes en los tejidos (Urquhart et al., 1996).
En los dos lechones sacrificados a los 10 días p.i. se encontró entre 2-16 esporas por animal dentro de los entericitos, posiblemente debido a que las esporas inoculadas no los lograron afectar, aunque también pudo deberse a que no se estudiaron las secciones adecuadas del tracto digestivo o porque no se estudiaron tejidos extra intestinales. Por otro lado, no se puede descartar que la coloración de plata no haya sido la más adecuada, toda vez que presenta resultados variables (Cook, 2000).

El estudio de los cortes histológicos del intestino, al final del ensayo, mostraron que la porción más infectada con esporas de $E$. bieneusi fue la porción media del yeyuno (Cuadro 1). Resultados similares se encontraron en el estudio de las distintas porciones del intestino de pacientes inmunodeficientes, donde el yeyuno fue la porción más afectada (Weber y Bryan, 1994).

Las lesiones histopatológicas encontradas correspondieron a atrofia de las vellosi- 
Cuadro 1. Número de esporas de Enterocytozoon bienensi en cortes histológicos de intestino en lechones (diagnóstico con coloración de plata)

\begin{tabular}{|c|c|c|c|c|c|}
\hline \multirow{2}{*}{ Cerdo } & \multicolumn{4}{|c|}{ Porción de intestino } & \multirow{2}{*}{ Total } \\
\hline & Duodeno & Yeyuno & Ileon & Colon & \\
\hline 1 & 5 & 8 & 3 & 0 & 16 \\
\hline 2 & 3 & 0 & 1 & 0 & 4 \\
\hline 3 & 0 & 2 & 0 & 5 & 7 \\
\hline 4 & 1 & 4 & 0 & 0 & 5 \\
\hline 5 & 1 & 1 & 0 & 0 & 2 \\
\hline 6 & 0 & 1 & 0 & 2 & 3 \\
\hline 7 & 1 & 3 & 3 & 1 & 8 \\
\hline 8 & 1 & 2 & 1 & 0 & 4 \\
\hline 9 & 1 & 2 & 0 & 1 & 4 \\
\hline Total & 13 & 23 & 8 & 9 & 53 \\
\hline
\end{tabular}

dades y presencia de polimorfos nucleares. Kotler et al. (1994) obtuvieron resultados similares en su estudio en pacientes con microsporidiosis. Sin embargo, no se observó ningún signo clínico como diarrea, vómito ni pérdida de peso en los animales. Este hecho puede indicar la importancia del genotipo de las esporas, ya que el inóculo del presente estudio provino de un niño con microsporidiosis. Los estudios moleculares determinarán si existe diferencia entre las esporas de E. bieneusi encontradas en humanos y cerdos.

Los análisis estadísticos encontraron 9 éxitos y 0 fracasos. La simulación se corrió considerando $\alpha_{1}=10$ y $\alpha_{2}=1$ y luego de 10000 iteracciones se encontró que el $90 \%$ de los valores de la probabilidad de infección estaban entre 0.741 y 0.9949 .

\section{Literatura Citada}

1. Atías A. 1991. Microsporidiosis. En: Parasitología clínica. $3^{\mathrm{a}}$ ed. Chile: Public. Téc. Mediterráneo. p 291-294.

2. Cama V, Pearson J, Cabrera L, Pacheci L, Gilman R, Meyer S, Ortega Y, Xiao L. 2007. Transmission of
Enterozytozoon bieneusi between a child and guinea pigs. J Clin Microbiol 45: 2708-2710.

3. Cook DJ, Pallister CJ. 1998. Cellular pathology. Oxford, UK: Ed. ButterworthHeinemann. 373 p.

4. Del Águila C, Rueda C, De la Camara C, Fenoy S. 2001. Seroprevalence of anti-Encephalitozoon antibodies in Spanish immunocompetent subjects. J Eukaryot Microbiol (Suppl.): 75S-78S.

5. Dengjel B, Zahler M, Hermanns W, Heinritzi K, Spillmann T, Thomschke A, Läscher T, et al. 2001. Zoonotic potential of Enterozytozoon bieneusi. J Clin Microbiol 39: 4495-4499.

6. Desplazes P, Mathis A, Weber R. 2000. Epidemiology and zoonotic aspects of microsporidia of mammals and birds. Contrib Microbiol 6: 236-260.

7. Desportes L, Charpentier LE, Galian $A$, Bernard F, Cochand-Priollet B, Lavengne A, Ravisse P, Modigliani $R$. 1985. Ocurrence of a new microsporidian: Enterocytozoon bieneusi n. G., n. $\mathrm{Sp}$., in the enterocytes of a human patient with AIDS. J Protozool 32: 250-254.

8. Didier E, Weiss L. 2006. Microsporidosis: current status. Curr Opin Infect Dis 19: 485-492. 
9. Isemberg H. 1998. Essential procedures for clinical microbiology. Washington: ASM Press. 898 p.

10. Kotler D, Giang T, Garro M, Orestein J. 1994. Light microscopic diagnosis of microsporidiosis in patients with AIDS. Am J Gastroenterol 89: 540-544.

11. Murray P, Kobayashi G, Pfaller P, Rosenthal K. 1997. Protozooarios intestinales y urogenitales. En: Microbiología médica. $2^{\mathrm{a}}$ ed. España: Harcourt Brace. p 460.

12. OPS. 2003. Zoonosis y enfermedades transmisibles comunes al hombre y a los animales. Volumen III: parasitosis. $3^{\mathrm{a}}$ ed. Washington: OPS. p 80-83.

13. Sandfort J, Hannemann A, Gelderblom H, Stark K, Owen R, Ruf B. 1994. Enterocytozoon bieneusi infection in an immunocompetent patient who had acute diarrhea and who was not infected with the human immunode- ficiency virus. Clin Infect Dis 19: 514516.

14. Schwartz D, Sobottka I, Cali A, Visvesvara R. 1996. Pathology of microsporidiosis: emerging parasitic infections in patients with acquired immunodeficiency syndrome. Arch Pathol Lab Med 120: 173-188.

15. Tzipori S, Kondova I, Mansfield K, Buckholt A, Stein B, Widmer B, Carville A, Lackner A. 1998. Transmission and serial propagation of Enterocytozoon bieneusi from humans and Rhesus macaques in gnotobiotic piglets. Infect Immun 66: 5515-5519.

16. Urquhart G, Armour J, Duncan J, Dunn A, Jennings F. 1996. Veterinary parasitology. $2^{\mathrm{a}}$ ed. Oxford, UK: Blackwell. 307 p.

17. Weber R, Bryan RT. 1994. Microsporidial infections in immunodeficient and immunocompetent patients. Clin Infect Dis 19: 517-521. 